\title{
Improving Duty Cycle-based MAC Protocol in Wireless Networks using AI and Machine Learning
}

\author{
S. S. Ponde, S. S. Lomte
}

\begin{abstract}
Duty cycle of a Medium Access Control (MAC) protocol is made up of sleep phase, wake-up phase and listen phase. MAC protocols usually proposes to optimize the duration of the wake-up and listen phases, in order to increase the duration of the sleep phase, thereby reducing the unwanted energy consumption of the wireless node. In this paper, we propose an Artificial Intelligence (AI) and machine learning (ML) based approach, which uses a hybrid combination of Time Division Multiple Access (TDMA), Bitmap Assisted MAC (BMA) and Sensor MAC (SMAC). The machine learning layer utilizes the duty cycle in the MAC layer, and generates multiple solutions for a given wireless communication. The AI layer then selects the best solution from the generated solutions by incorporating a duty cycle factor in the selection function, thereby optimizing the duty cycle of the protocol. The proposed system shows a 15\% improvement in communication speed, and a $10 \%$ reduction in energy consumption across multiple communications. We plan to further extend this work for rural India, and apply it to real time agricultural applications.
\end{abstract}

Keywords: Artificial Intelligence (AI), MAC, TDMA, BMA, SMAC, Duty cycle.

\section{INTRODUCTION}

Duty cycle controls the wake up, sleep and listen states of a node in the MAC protocol. Initially the node is in the sleep phase, during the sleep phase the node does not perform any communication activities, but waits for getting into listen phase. In the listen phase, the node consumes minimal power and listens for any packets which might match its signature [1]. If the packet's signature matches with the node, then it goes into wake up mode and performs operations according to the packet in picture, if the signature does not match it again goes into sleep mode for a particular number of clock cycles as decided by the MAC protocol [2].

Tuning the duty cycle carefully and making it optimum is a task of utmost importance when it comes to designing a MAC protocol. If the sleep phase is made too large, then the node

Revised Manuscript Received on December 30, 2019.

* Correspondence Author

S. S. Ponde*, Research Scholar, Deogiri Institute of Engineering and Management Studies, Aurangabad, Maharashtra, India. Email: smita.ponde@gmail.com

Dr. S. S. Lomte, Director, Radhai Mahavidyalaya, Aurangabad, Maharashtra, India. Email: drsantoshlomte@gmail.com

(C) The Authors. Published by Blue Eyes Intelligence Engineering and Sciences Publication (BEIESP). This is an open access article under the CC BY-NC-ND license (http://creativecommons.org/licenses/by-nc-nd/4.0/) might miss some packets, which will affect the packet delivery ratio and the throughput of the network, while a shorter sleep phase will toggle the node between listen and sleep phases rigorously[3] thereby increasing the power consumption of the node, and reducing the lifetime of the network. While sleep phase is of importance, the listen up phase must also be carefully planned [4]. A shorter listen cycle might reduce the successfully captured packet patterns, which might reduce the packet delivery ratio and affect the throughput too, while a longer listen cycle will increase the energy consumption exponentially, and the effect will be much larger as compared to that of a longer sleep phase [5]. Therefore, a carefully planned duty cycle is needed in order to optimize the sleep and listen phases. The wakeup phase is generally automatically optimized, because it starts when the packet signature is matched in the listen phase, and ends as soon as the packet reception is completed [6].

In this paper, we propose novel machine learning based MAC protocol which takes into consideration the duty cycle optimization of the nodes, so that wake up, listen and sleep phases are optimized. In the next section we describe various duty cycle optimization protocols, and their comparisons, followed by our Duty cycle based machine learning and AI based MAC protocol with localized learning, the paper concludes by the comparison of standard MAC protocol results with our optimized protocol. We also propose some future areas in which this work can be taken further in order to assist the node lifetime in agriculture industry.

\section{LITERATURE SURVEY}

Here we review various duty cycle aware protocols, which make sure that the sleep phases are given most of the cycle, while the awake phases are turned ON when needed [7]. This reduces the power consumption by many folds [8], and reduces latency in the network. Authors in [9] evaluate that the energy consumption is more in wake phase than sleep phase. WSN MAC protocols are either synchronous or asynchronous. Synchronous protocols have fixed wake and sleep cycles, while asynchronous ones have wake and sleep cycles either initiated by transmitter or receiver. Transmitter initiated approach is when the transmitter keeps on sending bursts of data whenever a sufficient amount of it is available, while receiver-initiated approach uses beaconing techniques. These approaches are further divided into multiple categories, some of which are described in this section.

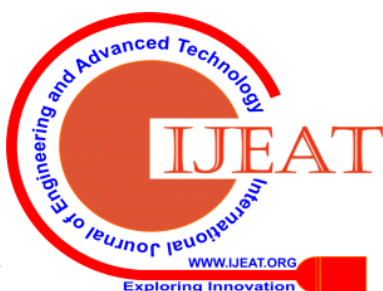




\section{A. Synchronous MAC with low duty cycles}

Every node generally has wake and sleep modes. Wake modes are transmission/reception modes while sleep modes are resting periods, where minimum amount of energy is consumed by the receiver and transmitter alike.

In [10], authors have introduced Power Aware Clustered TDMA (PACT) to employ passive aggregation, where network nodes perform power aware time division multiple access using a clustered approach. Nodes are classified into normal nodes, cluster heads and gateway nodes. Both gateway and heads interchange their duties in order to conserve power. Usually synchronization consumes a lot of power due to the fact that the nodes need to communicate with each other for higher level of stability in communication. Also, any node which has knowledge about the network can perform communication, which causes high delay and security issues as well. A WSN based MAC protocol named as Sensor MAC or S-MAC is proposed in [11]. It supports self-configuration and reduces energy consumption by tightly integrating the sleep and wake cycles [12]. SMAC assumes that applications are not-real time and can endure some latency if power efficiency is improved. This it cannot be used for applications which are delay-aware like high speed sensor applications. Thus, in this case, the nodes sleep and wake up as per the power requirement of the network and the neighbouring nodes. Therefore, nodes wake up based on the schedule given to them by their neighbours, and thus each neighbour always has some communication slots remaining for performing transmission and reception. Network can thus perform Request-To Send (RTS)/ Clear-To-Send (CTS) signalling as nodes can go from wake stage to idle stage to sleep stage. S-MAC uses bursts to send data between nodes [13]. Thus, there are a lot of fragmented packets, which leads to fragmentation of the network. Due to this fact the overall QoS parameters like delay, jitter, throughput and packet delivery ratio (PDR) are affected [14]. Due to the multi-hop nature of SMAC the fairness of the network can reduce and thus the network might not perform well in real time cases [15]. This increases the latency in the network. T-MAC [16] boosts the performance of S-MAC by introducing dynamic clock cycle arrangement rather than static one. Here the communication takes place in bursts of dynamic length, and thereby not making the network prone to delays and other issues. This also predicts the length of the variable packets by keeping a check on the observed packet sizes. T-MAC uses the RTS and CTS method. If RTS doesn't get CTS response then it tries out once more before going to next packet. Here the data is sent in one hop, thereby reducing the latency when compared to SMAC. Here the neighbour waits for some clock cycles before sending data or going to sleep. Due to which some of the QoS parameters are improved. RMAC [17] has 3 states in every communication cycle (SYNC, WAKE, and SLEEP). It uses a pioneer frame to show status to other nodes in the network. An upgradation to RMAC is called as P-MAC [18] which sends multiple packets per duty cycle and thus handles higher traffic than RMAC. This protocol performs node clustering around the receiver node, and thus uses receiver-based communication system. Clustered nodes maintain similar timings for communication which is differentiated by different clusters of the nodes. It uses pipelining for communication, and the clustering information is sent via RTS, to which CTS

responds in a continuous window, and thus performs synchronous communication.

\section{B. Asynchronous MAC protocols with low duty cycle}

Berkeley MAC a.k.a. B-MAC [19] is an asynchronous MAC protocol. In B-MAC, each node has independent programming cycles. Each node keeps on transmission of data enabled for a period longer than other node's sleep time, thereby all requested nodes will at least be ON for one cycle to get the request of this transmitting node. Once the node gets the data, it checks the pre-amble bits, and goes into sleep or stays awake based on the addressing. The major drawback is overhearing, which can be reduced, but due to this the overall network QoS is improved. X-MAC [20] reduces these drawbacks by keeping preambles as short as possible. The short preamble only contains target address, thereby reducing the delay in communication, and also helping the non-targeted nodes to go into sleep mode as early as possible [21]. The major issue due to short pre-ambles is the inability to reconfigure the nodes once they have been configured. Also, due to short preambles, no traffic information is sent, thereby causing unwanted collisions in the network. Thereby the traffic is impacted by this protocol. RI-MAC [22] is based on received initiation, which turns $\mathrm{ON}$ the transmission only when receiver is ready to get the data, or is in WAKE mode. It uses similar strategies to XMAC and BMAC but waits for received initiation to start and stop the wake and sleep cycles. Here receiver uses beaconing to inform nodes about its status. Apart from MAC protocols, node's quality and other parameters also have an impact on the network's lifetime and communication quality [23]. Static WSNs are not affected by these parameters. DiS-MAC [24] was made for single direction traffic, majorly used by authorities in order to perform communication. Due to the high-power consumption nature of this approach, it is generally not used for multi-direction communication systems, approaches like LC-MAC [24] and WiFi-MAC [25] perform this task. While techniques like CSMA/CA [26] and DiS-MAC are not used in important applications. In [27] it is observed that perfect asynchronization produces low delay and energy, but this is flawed, due to the fact that in the case of [27], there are higher packet drops which reduces PDR, increases re-transmissions and therefore reduces the overall QoS. MAC systems must provide tradeoffs between the techniques and make the network efficient. Therefore, our new work is proposing a machine learning and AI based MAC layer which increases the network QoS and does not compromise on any of the network primary performance parameters. This technique is given in the next section.

\section{PROPOSED METHODOLOGY}

\section{Duty Cycle-Based Machine Learning And Ai Based Mac With Localized Learning (Dcmlaillmac)}

Machine learning has been a state-of-the-art method for optimizing complex problems. In our case, the optimization problem converges to selecting the best route, with the most optimum duty cycle structure and the best MAC protocol. The machine learning layer also uses an AI selection unit which selects the best combination of methods in order to optimize the overall protocol.

The proposed technique can be described as follows,

Input parameters,

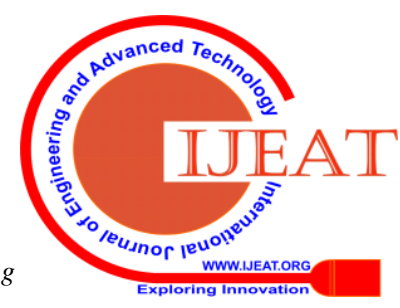


1. Number of solutions (Ns)

2. Number of rounds $(\mathrm{Nr})$

3. Learning convergence (Lc)

4. Max nodes per solution (Nmax)

5. Link quality (Lq)

6. Max Wake Cycle (MWC)

7. Max Sleep Cycle (MSC)

8. Max Listen Cycle (MLC)

Algorithm,

1. Select a source and destination src and dest

2. Find the reference distance between the nodes (dref)

3. For each solution,

a. Find the number of nodes

$(\mathrm{N})=$ random value $*$ Nmax

b. Select $\mathrm{N}$ random nodes between source and destination, meeting the following condition, dis + did $>=$ dref

and, dis $<$ dref $\&$ did $<$ dref

where, dis = Distance between the current node and source

did $=$ Distance between the current node and destination

c. Evaluate the Learning Metric(LM),

$\mathrm{LM}=\operatorname{Sum}(\mathrm{Di}, \mathrm{i}-1 / \mathrm{Ei}-1+1 / \mathrm{LQ}(\mathrm{i}, \mathrm{i}-1)) * \mathrm{CF}$

where,

Di,i-1 = Distance between the selected nodes i and i-1

Ei-1 = Energy of the i-1th node

LQ(i,i-1) = Link quality between ith and i-1th node

$\mathrm{CF}=$ Cycle factor, the value of the cycle factor is given as,

$\mathrm{CF}=1$, when Cycle Factor Constant $(\mathrm{CFC})=1$

$\mathrm{CF}=1 / \mathrm{CFC}$, when $\mathrm{CFC}<1$

$\mathrm{CF}=\mathrm{CFC}$, when $\mathrm{CFC}>1$

here, $\mathrm{CFC}$ or cycle factor constant is formulized as

follows,

$\mathrm{CFC}=0.7 *(\operatorname{RAND}(0, \mathrm{MWC}))+0.2 *(\operatorname{RAND}(0, \mathrm{MLC}))$ $+0.1$

* (RAND $(0$, MSC $))$

The weights are decided based on how we need the duty cycle to vary. In our case, we need the system to listen for $20 \%$ of the cycle, sleep for $10 \%$ of the cycle and wake for $70 \%$ of the cycle (when communication is needed)

If the value of CFC is closer to 1, that means the duty cycle is good enough, and we select the given values of sleep, wakeup and listen timings, in order to optimize the communication cycle.

4. Find the mean of all the LQ values (LMean), and then evaluate learning threshold (Lth), as follows,

Lth $=$ LMean $*$ Lc

5. Pass all solutions where $\mathrm{LM}<$ Lth to next iteration, and modify all the other solutions

6. Repeat steps 2 to 5 for all Nr rounds

7. At the end of the Nrth round, select the solution with minimum value of LM (Smin)

8. Select a random MAC implementation from TDMA, BMA or SMAC for transmission of data on the path selected by Smin

9. Store this information in a table for learning.

For a new communication between the nodes, refer the table and select the MAC protocol which gives minimal value of LM, thereby optimizing the distance of communication, the energy of communication and the link quality for communication. These 3 parameters combined affect the output QoS of the system, and optimize the end to end delay, jitter in delay, the packet delivery ratio (PDR) and communication throughput. Our algorithm is compared with TDMA, SMAC and BMAC, and results are mentioned in the next section under various network scenarios.

\section{RESULT AND DISCUSSION}

Our protocol was tested and trained under different network conditions, and the results were evaluated for various communication combinations. This is done in order to evaluate the network for various scenarios. Following table shows the network parameters.

Table- I: Network Parameters

\begin{tabular}{|l|l|}
\hline Parameter & Value \\
\hline Routing algorithm & AODV \\
\hline Quantity of nodes & 30 to 100 \\
\hline Network type & MANET \\
\hline Queue & Priority drop tail \\
\hline Network size & $300 \mathrm{~m}$ x $300 \mathrm{~m}$ \\
\hline MAC Type & 802.11 \\
\hline No. of communications & $1-10$ \\
\hline
\end{tabular}

A sample machine learning table obtained from our simulations with 40 solutions and 20 iterations is shown in Table II.

From this table, we select the 35th solution, which has the minimum LM value obtained at the 15th iteration, and use BMA for communication with the obtained parameter values. In our simulations, we varied the number of solutions from 10 to 200, and the number of iterations from 20 to 500, and observed that the optimum solution for 100 nodes is obtained around 105 solutions and 472 iteration rounds. Any number of solutions and iterations more than that, does not give any significant improvement in the network performance of the system.

In our analysis, we compared the end to end delay for communication, the energy needed for communication, communication throughput, packet delivery ratio and the communication delay jitter of the proposed protocol with an AI based protocol which does not consider duty cycle, the TDMA protocol, BMA protocol and SMAC protocol. 
Improving Duty Cycle-based MAC Protocol in Wireless Networks using AI and Machine Learning

Table- II: Example LUTs of the Machine Learning Algorithm

\begin{tabular}{|c|c|c|c|c|c|c|}
\hline Solution & LM & MAC & Sleep Tims & Listen Tim & Wake Tim & Iteration \\
\hline 1 & 2.57 & TDMA & 0.15 & 0.19 & 0.66 & 1 \\
\hline 2 & 2.66 & TDMA & 0.1 & 0.1 & 0.8 & 13 \\
\hline 3 & 2.17 & SMAC & 0.11 & 0.19 & 0.7 & 18 \\
\hline 4 & 2.38 & BMA & 0.11 & 0.23 & 0.66 & 5 \\
\hline 5 & 2.94 & BMA & 0.13 & 0.26 & 0.61 & 13 \\
\hline 6 & 2.18 & SMAC & 0.1 & 0.18 & 0.72 & 18 \\
\hline 7 & 2.05 & BMA & 0.06 & 0.03 & 0.91 & 1 \\
\hline 8 & 2.33 & BMA & 0.14 & 0.04 & 0.82 & 19 \\
\hline 9 & 2.19 & BMA & 0.17 & 0.09 & 0.74 & 1 \\
\hline 10 & 2.14 & TDMA & 0.07 & 0.16 & 0.77 & 17 \\
\hline 11 & 2.09 & SMAC & 0.12 & 0.08 & 0.8 & 18 \\
\hline 12 & 2.04 & TDMA & 0.18 & 0.21 & 0.61 & 16 \\
\hline 13 & 2.93 & BMA & 0.06 & 0.2 & 0.74 & 13 \\
\hline 14 & 2.47 & SMAC & 0.15 & 0.16 & 0.69 & 14 \\
\hline 15 & 2.66 & SMAC & 0.04 & 0.26 & 0.7 & 17 \\
\hline 16 & 2.48 & TDMA & 0.2 & 0.11 & 0.69 & 4 \\
\hline 17 & 2.21 & TDMA & 0.07 & 0.26 & 0.67 & 19 \\
\hline 18 & 2.67 & BMA & 0.03 & 0.03 & 0.94 & 4 \\
\hline 19 & 2.34 & SMAC & 0.05 & 0.02 & 0.93 & 3 \\
\hline 20 & 2.28 & SMAC & 0.11 & 0.09 & 0.8 & 12 \\
\hline 21 & 2.38 & SMAC & 0.04 & 0.02 & 0.94 & 11 \\
\hline 22 & 2.38 & BMA & 0.06 & 0.3 & 0.64 & 9 \\
\hline 23 & 2.47 & BMA & 0.07 & 0.18 & 0.75 & 19 \\
\hline 24 & 2.38 & BMA & 0.06 & 0.2 & 0.74 & 20 \\
\hline 25 & 2.10 & TDMA & 0.08 & 0.23 & 0.69 & 20 \\
\hline 26 & 2.31 & SMAC & 0.2 & 0.07 & 0.73 & 13 \\
\hline 27 & 2.17 & TDMA & 0.14 & 0.3 & 0.56 & 14 \\
\hline 28 & 2.23 & BMA & 0.16 & 0.11 & 0.73 & 16 \\
\hline 29 & 2.17 & SMAC & 0.09 & 0.27 & 0.64 & 1 \\
\hline 30 & 2.15 & SMAC & 0.03 & 0.04 & 0.93 & 12 \\
\hline 31 & 2.12 & TDMA & 0.13 & 0.17 & 0.7 & 12 \\
\hline 32 & 2.10 & TDMA & 0.08 & 0.19 & 0.73 & 4 \\
\hline 33 & 2.08 & SMAC & 0.08 & 0.07 & 0.85 & 2 \\
\hline 34 & 2.05 & BMA & 0.14 & 0.13 & 0.73 & 15 \\
\hline 35 & 2.03 & BMA & 0.15 & 0.14 & 0.71 & 15 \\
\hline 36 & 2.55 & BMA & 0.2 & 0.13 & 0.67 & 13 \\
\hline 37 & 2.68 & TDMA & 0.05 & 0.2 & 0.75 & 6 \\
\hline 38 & 2.42 & SMAC & 0.19 & 0.16 & 0.65 & 14 \\
\hline 39 & 2.94 & TDMA & 0.17 & 0.1 & 0.73 & 3 \\
\hline 40 & 2.08 & BMA & 0.08 & 0.25 & 0.67 & 1 \\
\hline
\end{tabular}

The following Table III (a) and Table III (b) shows Energy Consumption obtained for 30 nodes and 40 nodes.

Table- III (a): Energy consumption for 30 nodes

\begin{tabular}{|r|r|r|r|r|r|}
\hline No. of coms. & E TDMA (m) $)$ & E BMA (mJ) & ESMAC (m) $)$ & EAl w/o Duty Cycle (m) & EAl with Duty Cycle (mJ) \\
\hline 1.00 & 17.73 & 8.40 & 5.60 & 2.80 & 0.93 \\
\hline 2.00 & 25.20 & 14.93 & 15.87 & 4.67 & 2.80 \\
\hline 3.00 & 45.73 & 20.53 & 16.80 & 7.47 & 2.80 \\
\hline 4.00 & 56.93 & 24.27 & 33.60 & 9.33 & 7.47 \\
\hline 5.00 & 52.27 & 35.47 & 23.33 & 13.07 & 6.53 \\
\hline 6.00 & 108.27 & 67.20 & 31.73 & 7.47 & 6.53 \\
\hline 7.00 & 68.13 & 74.67 & 32.67 & 7.47 & 13.07 \\
\hline 8.00 & 107.33 & 39.20 & 51.33 & 22.40 & 8.40 \\
\hline 9.00 & 84.93 & 91.47 & 67.20 & 13.07 & 9.33 \\
\hline 10.00 & 154.93 & 73.73 & 62.53 & 14.00 & 17.73 \\
\hline 15.00 & 191.33 & 164.27 & 121.33 & 21.47 & 20.53 \\
\hline 20.00 & 337.87 & 184.80 & 165.20 & 36.40 & 23.33 \\
\hline 25.00 & 350.00 & 277.20 & 223.07 & 36.40 & 30.80 \\
\hline 50.00 & 661.73 & 503.07 & 308.93 & 73.73 & 91.47 \\
\hline 75.00 & 1374.80 & 358.40 & 597.33 & 108.27 & 89.60 \\
\hline 100.00 & 1157.33 & 515.20 & 717.73 & 162.40 & 119.47 \\
\hline
\end{tabular}

Table- III (b): Energy consumption for 40 nodes

\begin{tabular}{|r|r|r|r|r|r|}
\hline No. of coms. & E TDMA (m) & E BMA (m) & ESMAC (m) & EAl w/o Duty Cycle (m) & EAl with Duty Cycle (m) \\
\hline 1 & 22.40 & 8.40 & 12.13 & 3.73 & 1.87 \\
\hline 2 & 25.20 & 13.07 & 16.80 & 6.53 & 3.73 \\
\hline 3 & 70.00 & 27.07 & 26.13 & 5.60 & 3.73 \\
\hline 4 & 82.13 & 27.07 & 45.73 & 12.13 & 6.53 \\
\hline 5 & 79.33 & 42.00 & 56.00 & 17.73 & 12.13 \\
\hline 6 & 129.73 & 37.33 & 38.27 & 17.73 & 14.93 \\
\hline 7 & 144.67 & 45.73 & 60.67 & 16.80 & 11.20 \\
\hline 8 & 154.00 & 57.87 & 83.07 & 23.33 & 16.80 \\
\hline 9 & 132.53 & 85.87 & 85.87 & 31.73 & 16.80 \\
\hline 10 & 187.60 & 112.93 & 124.13 & 32.67 & 22.40 \\
\hline 15 & 232.40 & 159.60 & 100.80 & 37.33 & 19.60 \\
\hline 20 & 475.07 & 271.60 & 129.73 & 61.60 & 37.33 \\
\hline 25 & 414.40 & 313.60 & 258.53 & 34.53 & 54.13 \\
\hline 50 & 863.33 & 690.67 & 421.87 & 77.47 & 118.53 \\
\hline 75 & 1217.07 & 921.20 & 511.47 & 257.60 & 124.13 \\
\hline 100 & 1410.27 & 1302.93 & 1209.60 & 201.60 & 174.53 \\
\hline
\end{tabular}

From the tables and the graphs, it is evident that TDMA consumes the most energy, while our AI based protocol consumes least energy while communicating in the network. Our analysis show that the network lifetime is improved by more than $60 \%$ when compared to the AI based MAC protocol which does not consider duty cycle for optimization, and can be seen as follows,

\section{Lifetime improvement}

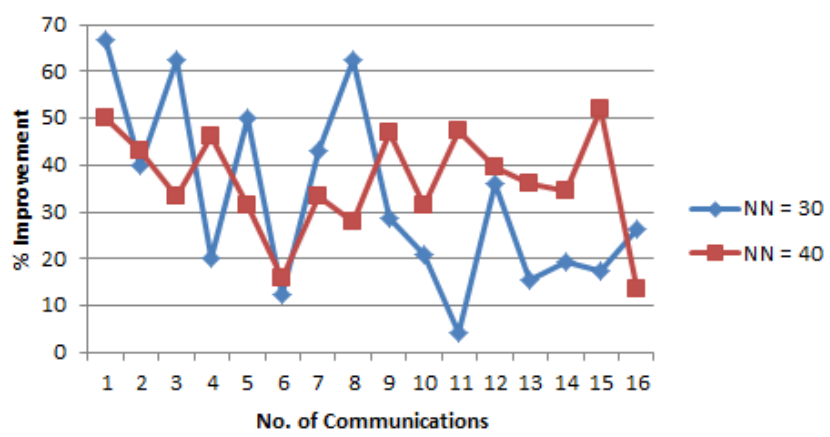

Fig. 1. Improvement in network lifetime

Similar comparison is made for end to end delay, which can be observed from the following table,

Table- IV (a): End to End delay for 30 nodes

\begin{tabular}{|r|r|r|r|r|r|}
\hline No. of coms. & D TDMA (ms) & D BMA (ms) & D SMAC (ms) & D Al w/o Duty Cycle (ms) & D Al with Duty Cycle (ms) \\
\hline 1 & 0.16 & 0.07 & 0.05 & 0.02 & 0.01 \\
\hline 2 & 0.25 & 0.12 & 0.09 & 0.05 & 0.04 \\
\hline 3 & 0.30 & 0.31 & 0.14 & 0.08 & 0.04 \\
\hline 4 & 0.37 & 0.23 & 0.35 & 0.07 & 0.06 \\
\hline 5 & 0.71 & 0.42 & 0.23 & 0.06 & 0.09 \\
\hline 6 & 0.60 & 0.56 & 0.51 & 0.11 & 0.10 \\
\hline 7 & 0.89 & 0.62 & 0.49 & 0.16 & 0.13 \\
\hline 8 & 0.87 & 0.50 & 0.44 & 0.09 & 0.10 \\
\hline 9 & 0.95 & 0.49 & 0.63 & 0.13 & 0.15 \\
\hline 10 & 1.57 & 0.81 & 0.71 & 0.27 & 0.09 \\
\hline 15 & 1.59 & 0.89 & 1.10 & 0.18 & 0.28 \\
\hline 20 & 2.03 & 1.89 & 1.11 & 0.22 & 0.36 \\
\hline 25 & 3.24 & 2.45 & 1.95 & 0.45 & 0.42 \\
\hline 50 & 5.08 & 3.73 & 3.73 & 0.60 & 0.82 \\
\hline 75 & 8.22 & 3.52 & 3.97 & 1.19 & 1.02 \\
\hline 100 & 11.41 & 10.11 & 6.20 & 2.35 & 1.68 \\
\hline
\end{tabular}


Table- IV (b): End to End delay for 40 nodes

\begin{tabular}{|r|r|r|r|r|r|}
\hline No. of coms. & D TDMA (ms) & D BMA (ms) & D SMAC (ms) & D Al w/o Duty Cycle (ms) & D Al with Duty Cycle (ms) \\
\hline 1 & 0.15 & 0.10 & 0.11 & 0.04 & 0.02 \\
\hline 2 & 0.29 & 0.24 & 0.17 & 0.03 & 0.03 \\
\hline 3 & 0.63 & 0.33 & 0.20 & 0.11 & 0.05 \\
\hline 4 & 0.78 & 0.54 & 0.37 & 0.15 & 0.07 \\
\hline 5 & 0.72 & 0.61 & 0.36 & 0.16 & 0.07 \\
\hline 6 & 1.29 & 0.45 & 0.46 & 0.08 & 0.15 \\
\hline 7 & 1.47 & 0.63 & 0.67 & 0.12 & 0.15 \\
\hline 8 & 1.23 & 0.97 & 0.55 & 0.16 & 0.20 \\
\hline 9 & 1.51 & 1.33 & 0.84 & 0.19 & 0.11 \\
\hline 10 & 2.45 & 1.21 & 0.88 & 0.14 & 0.24 \\
\hline 15 & 1.87 & 1.45 & 1.35 & 0.55 & 0.30 \\
\hline 20 & 3.38 & 1.35 & 1.92 & 0.73 & 0.44 \\
\hline 25 & 4.66 & 3.02 & 2.66 & 0.68 & 0.60 \\
\hline 50 & 10.33 & 6.93 & 5.88 & 1.83 & 1.04 \\
\hline 75 & 18.28 & 11.12 & 5.59 & 1.26 & 1.11 \\
\hline 100 & 13.47 & 9.39 & 8.70 & 2.18 & 1.19 \\
\hline
\end{tabular}

The end to end delay follows the same trend as the energy consumption, and improves the overall speed of the MAC protocol when AI is used. The speed improvement guarantees faster response of the system, and good throughput when compared with all the other MAC protocols. The comparison graph of delay improvement shows a 50\% increase in system speed when compared with the AI based MAC protocol without duty cycle optimization.

\section{Speed improvement v/s Num. Comms.}

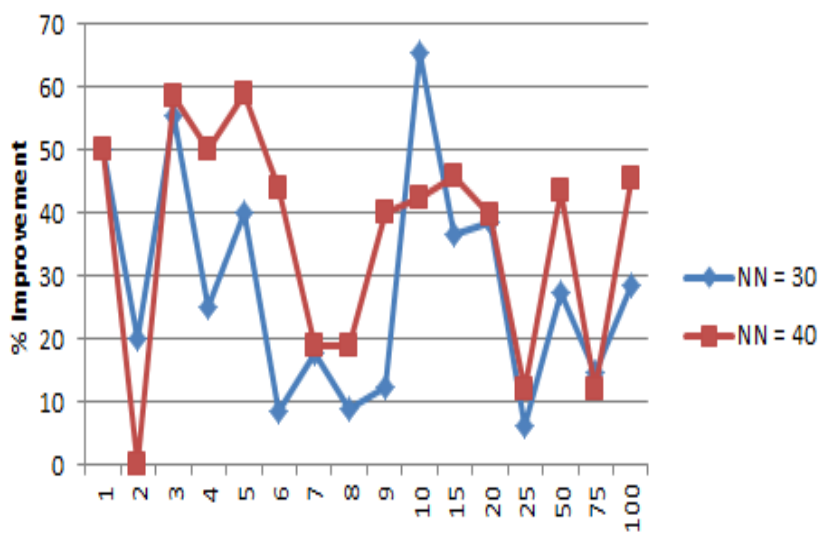

No. of Communications

Fig. 2. Improvement in overall network speed

The packet delivery ratio (PDR) is also improved when compared to BMA and SMAC, and has similar values when compared to TDMA. TDMA being a low complexity protocol, has minimal or no loss in packets, while BMA and SMAC do. Our AI based algorithm overcomes those issues with BMA and SMAC in order to match the PDR better than TDMA, moreover it improves the PDR as reported by the AI based MAC protocol without duty cycle consideration. The results for the same can be observed from the following tables,
Table- V (a): PDR for 30 nodes

\begin{tabular}{|r|r|r|r|r|r|}
\hline No. of coms. & PDR TDMA (\%) & PDR BMA (\%) & PDR SMAC (\%) & PDR Al w/o Duty Cycle (\%) & PDR Al with Duty Cycle (\%) \\
\hline 1 & 96.57 & 92.00 & 94.00 & 96.00 & 95.00 \\
\hline 2 & 95.31 & 95.00 & 95.50 & 94.50 & 95.50 \\
\hline 3 & 96.13 & 94.00 & 93.00 & 95.67 & 95.33 \\
\hline 4 & 98.02 & 90.75 & 93.50 & 97.75 & 96.75 \\
\hline 5 & 94.76 & 94.60 & 93.60 & 94.40 & 96.00 \\
\hline 6 & 95.04 & 94.00 & 92.33 & 94.83 & 96.83 \\
\hline 7 & 97.24 & 92.29 & 92.43 & 96.71 & 95.14 \\
\hline 8 & 97.28 & 92.88 & 92.50 & 96.63 & 96.13 \\
\hline 9 & 95.41 & 93.56 & 96.00 & 95.00 & 98.89 \\
\hline 10 & 98.98 & 93.60 & 93.90 & 98.60 & 98.60 \\
\hline 15 & 96.13 & 95.60 & 95.20 & 95.47 & 97.47 \\
\hline 20 & 95.00 & 95.65 & 95.55 & 94.95 & 97.90 \\
\hline 25 & 94.73 & 92.84 & 93.96 & 94.20 & 96.44 \\
\hline 50 & 96.06 & 91.82 & 95.44 & 95.80 & 97.64 \\
\hline 75 & 95.75 & 92.91 & 93.32 & 95.09 & 97.11 \\
\hline 100 & 97.25 & 90.34 & 94.50 & 97.10 & 95.61 \\
\hline
\end{tabular}

Table- V (b): PDR for 40 nodes

\begin{tabular}{|r|r|r|r|r|r|}
\hline No. of coms. & PDR TDMA (\%) & PDR BMA (\%) & PDR SMAC (\%) & PDR Al w/O Duty Cycle (\%) & PDR Al with Duty Cycle (\%) \\
\hline 1 & 99.98 & 93.75 & 92.25 & 99.00 & 97.50 \\
\hline 2 & 94.25 & 91.13 & 92.63 & 94.13 & 97.13 \\
\hline 3 & 94.61 & 93.75 & 93.25 & 94.25 & 96.25 \\
\hline 4 & 96.64 & 94.88 & 92.63 & 96.38 & 95.63 \\
\hline 5 & 95.99 & 95.55 & 96.00 & 95.40 & 95.55 \\
\hline 6 & 97.58 & 91.38 & 94.75 & 96.88 & 96.50 \\
\hline 7 & 99.42 & 94.50 & 92.36 & 98.68 & 95.36 \\
\hline 8 & 95.57 & 95.06 & 94.78 & 94.69 & 95.06 \\
\hline 9 & 97.24 & 94.83 & 93.58 & 96.50 & 96.58 \\
\hline 10 & 97.46 & 96.38 & 95.18 & 97.28 & 98.03 \\
\hline 15 & 97.02 & 90.70 & 93.15 & 96.30 & 95.25 \\
\hline 20 & 96.36 & 93.94 & 95.18 & 95.40 & 98.70 \\
\hline 25 & 97.44 & 95.04 & 95.73 & 97.38 & 97.02 \\
\hline 50 & 97.30 & 95.16 & 93.53 & 97.29 & 95.93 \\
\hline 75 & 95.77 & 95.29 & 93.63 & 95.11 & 98.50 \\
\hline 100 & 98.77 & 94.34 & 95.49 & 98.57 & 95.26 \\
\hline
\end{tabular}

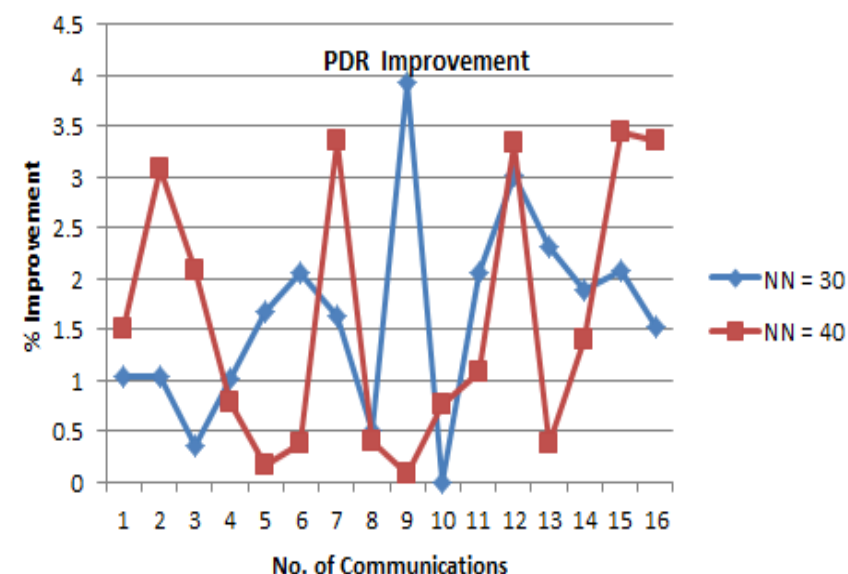

Fig. 3. PDR comparison

The other parameters follow this same trend and tend to improve the overall performance of the system even if the number of communications and other network parameters are varied. 


\section{CONCLUSION}

From the results we can observe that the QoS of the DC-ML-Ai-LL-MAC system is superior to the current state-of-the art standard MAC protocols like SMAC, BMA and TDMA and also from the non-duty cycle aware AI based MAC protocol. The AI layer also improves the overall speed in communication for the network and keeps a high packet delivery ratio for any type of network scenario. The proposed technique other techniques for any kind of network scenario and overall network QoS is also improved

\section{FUTURE WORK}

The proposed protocol demonstrates good quality of network QoS when compared with other standard protocols, and thus can be used for any real life wireless application. In future, we plan to apply this optimization to agriculture based networks, thereby assisting the farmers to get a better yield using modern day wireless networks.

\section{REFERENCES}

1. H. Luo, M. He, Z. Ruan and F. Chen, "A Duty-Cycle MAC Algorithm with Traffic Prediction for Wireless Sensor Networks," 2017 4th International Conference on Information Science and Control Engineering (ICISCE), Changsha, 2017, pp. 16-19.

2. Y. Liu et al., "QTSAC: An Energy-Efficient MAC Protocol for Delay Minimization in Wireless Sensor Networks," in IEEE Access, vol. 6 pp. 8273-8291, 2018.

3. S. Siddiqui, S. Ghani and A. A. Khan, "ADP-MAC: An Adaptive and Dynamic Polling-Based MAC Protocol for Wireless Sensor Networks," in IEEE Sensors Journal, vol. 18, no. 2, pp. 860-874, 15 Jan.15, 2018.

4. N. H. Bidoki, M. B. Baghdadabad, G. Sukthankar and D. Turgut, "Joint Value of Information and Energy Aware Sleep Scheduling in Wireless Sensor Networks: A Linear Programming Approach," 2018 IEEE International Conference on Communications (ICC), Kansas City, MO, 2018, pp. 1-6.

5. H. P. Gupta, S. V. Rao and T. Venkatesh, "Sleep Scheduling Protocol for \$k\$-Coverage of Three-Dimensional Heterogeneous WSNs," in IEEE Transactions on Vehicular Technology, vol. 65, no. 10, pp. 8423-8431, Oct. 2016.

6. Zhang Z., Shu L., Zhu C., Mukherjee M. (2018) A Short Review on Sleep Scheduling Mechanism in Wireless Sensor Networks. In: Wang L., Qiu T., Zhao W. (eds) Quality, Reliability, Security and Robustness in Heterogeneous Systems. QShine 2017. Lecture Notes of the Institute for Computer Sciences, Social Informatics and Telecommunications Engineering, vol 234. Springer, Cham

7. M. I. Khalil, M. A. Hossain, R. Mamtaz, I. Ahmed and M. Akter, "Time Efficient Receiver Oriented Sleep Scheduling for Underwater Sensor Network," 2017 IEEE International Conference on Imaging, Vision \& Pattern Recognition (icIVPR), Dhaka, 2017, pp. 1-6.

8. T. A. Al-Janabi and H. S. Al-Raweshidy, "An Energy Efficient Hybrid MAC Protocol With Dynamic Sleep-Based Scheduling for High Density IoT Networks," in IEEE Internet of Things Journal, vol. 6, no. 2, pp. 2273-2287, April 2019.

9. V. R. K. Ramachandran, D. V. Le, N. Meratnia and P. J. M. Havinga, "DiNAMAC: A disruption tolerant, reinforcement learning-based Mac protocol for implantable body sensor networks," 2017 IEEE SmartWorld, Ubiquitous Intelligence \& Computing, Advanced \& Trusted Computed, Scalable Computing \& Communications, Cloud \& Big Data Computing, Internet of People and Smart City Innovation (SmartWorld/SCALCOM/UIC/ATC/CBDCom/IOP/SCI), San Francisco, CA, 2017, pp. 1-9.

10. Pei, Guangyu \& Chien, C. (2001). Low power TDMA in large wireless sensor networks. 1. 347 - 351 vol.1. 10.1109/MILCOM.2001.985817.

11. Wei Ye, J. Heidemann and D. Estrin, "An energy-efficient MAC protocol for wireless sensor networks," Proceedings.Twenty-First Annual Joint Conference of the IEEE Computer and Communications Societies, New York, NY, USA, 2002, pp. 1567-1576 vol.3.

12. D. Kim, J. Jung, Y. Koo and Y. Yi, "Revisiting Sensor MAC for Periodic Monitoring: Why Should Transmitters Be Early Birds?," 2017 14th Annual IEEE International Conference on Sensing, Communication, and Networking (SECON), San Diego, CA, 2017, pp. 1-9.
13. M. Anwander, G. Wagenknecht, T. Braun and K. Dolfus, "BEAM: A Burst-aware Energy-efficient Adaptive MAC protocol for Wireless Sensor Networks," 2010 Seventh International Conference on Networked Sensing Systems (INSS), Kassel, 2010, pp. 195-202.

14. G. Corbellini, E. C. Strinati and A. Duda, "LA-MAC: Low-latency asynchronous MAC for wireless sensor networks," 2012 IEEE 23rd International Symposium on Personal, Indoor and Mobile Radio Communications - (PIMRC), Sydney, NSW, 2012, pp. 380-386.

15. F. D. Cunha, R. A. F. Mini and A. A. F. Loureiro, "Sensor-MAC with Dynamic Duty Cycle in wireless sensor networks," 2012 IEEE Global Communications Conference (GLOBECOM), Anaheim, CA, 2012, pp. 530-536.

16. Y. Zatout, E. Campo and J. Llibre, "T-TMAC: Energy Aware Sensor MAC Protocol for Health-Care Monitoring," 2012 IEEE Vehicular Technology Conference (VTC Fall), Quebec City, QC, 2012, pp. 1-5.

17. S. Yessad, F. Nait-Abdesselam, T. Taleb and B. Bensaou, "R-MAC Reservation Medium Access Control Protocol for Wireless Sensor Networks," 32nd IEEE Conference on Local Computer Networks (LCN 2007), Dublin, 2007, pp. 719-724

18. N. P. Khan and C. Boncelet, "PMAC: Energy Efficient Medium Access Control Protocol for Wireless Sensor Networks," MILCOM 2006 - 2006 IEEE Military Communications conference, Washington, DC, 2006, pp. 1-5.

19. R. V. Steiner, T. R. Mück and A. A. Fröhlich, "C-MAC: A configurable medium access control protocol for sensor networks," SENSORS, 2010 IEEE, Kona, HI, 2010, pp. 845-848.

20. A. Ullah, J. Ahn and Gayoung Kim, "X-MAC protocol with Collision Avoidance algorithm," 2013 Fifth International Conference on Ubiquitous and Future Networks (ICUFN), Da Nang, 2013, pp. 228-233.

21. Yomo, Hiroyuki \& Nguyen, Huan \& Kyritsi, Persefoni \& Duc Nguyen, Tien \& S. Chakraborty, Shyam \& Prasad, Ramjee. (2015). PHY and MAC performance evaluation of IEEE 802.11a WLAN over fading channels. IETE Journal of Research. 51. 10.1080/03772063.2005.11416381.

22. Y. Peng, Z. Li, W. Zhang and D. Qiao, "A lifetime-balancing MAC protocol under the end-to-end delay requirement," in Journal of Communications and Networks, vol. 19, no. 1, pp. 51-64, February 2017.

23. V. Potdar, A. Sharif and E. Chang, "Wireless Sensor Networks: A Survey," 2009 International Conference on Advanced Information Networking and Applications Workshops, Bradford, 2009, pp. 636-641.

24. T. Karveli, K. Voulgaris, M. Ghavami and A. H. Aghvami, DiS-MAC: A MAC protocol for sensor networks used for roadside and highway monitoring," 2009 International Conference on Ultra Modern Telecommunications \& Workshops, St. Petersburg, 2009, pp. 1-6.

25. Z. Zhang, W. P. Richard and A. Boukerche, "A fast MAC layer handoff protocol for WiFi-based wireless networks," IEEE Local Computer Network Conference, Denver, CO, 2010, pp. 684-690.

26. S. U. Rehman, S. Berber and A. Swain, "Performance analysis of CSMA/CA algorithm for wireless sensor network," TENCON 2010 2010 IEEE Region 10 Conference, Fukuoka, 2010, pp. 2012-2017.

27. S. Wang, J. Liu, S. Zhou, L. Zhou, M. Yin and H. Hao, "Cooperative relay MAC protocol for ad hoc networks," 2017 IEEE 17th International Conference on Communication Technology (ICCT), Chengdu, 2017, pp. 612-616.

\section{AUTHORS PROFILE}

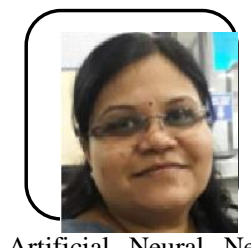

Smita Ponde received the Master of Technology degree in computer science from Dr. B. A. M University, Aurangabad (MH), India, in 2009. She is currently working toward his Ph.D. degree in the department of Computer Science, Dr. B. A. M University, Aurangabad, (MS), India. Her research interests include Wireless Sensor Networks, Artificial Neural Networks, Machine Learning and Data Mining. In particular her research focuses on development of Energy-Efficient MAC Protocols for Wireless Sensor Networks using machine learning and AI. Her papers have been published in various conferences and journals such as Springer, IEEE, IJSEM, IJERD, IJSER

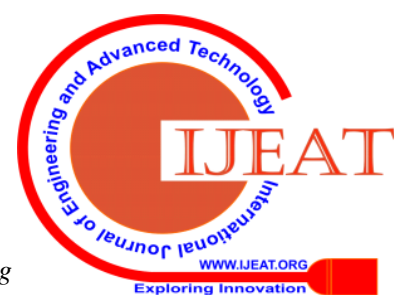


Dr. Santosh Lomte received the Master of Applied Science in Computer from Marathwada University, Aurabgabad (MS), India, M.E. Computer Science and Engineering from Dr. B.A.M.University, Aurangabad. He received a Ph.D in Computer Science with research area "Computer Programming In Operations Research -Simulation Based Techniques in 2010. He has published more than 35 research paper in various reputed Journals. 